\title{
Successful Postoperative Management of Gastrointestinal Stromal Tumor in a Dog
}

\section{Ki-Sung Lee ${ }^{1, t}$ \\ Keon Kim ${ }^{1, t}$ \\ Chul-Ho Yang ${ }^{2}$ \\ Guk-Hyun Suh ${ }^{1, *}$ \\ Chang-Min Lee ${ }^{1, *}$}

'Department of Veterinary Internal Medicine, College of Veterinary Medicine and BK21 FOUR program, Chonnam National University, Gwangju 61186, Korea

${ }^{2}$ Times Animal Medical Center, Suwon 16487, Korea
${ }^{\dagger} \mathrm{Ki}$-Sung Lee and Keon Kim contributed equally to this work.

*Correspondence: ghsuh@jnu.ac.kr (GukHyun Suh), cmlee1122@jnu.ac.kr (Chang-Min Lee)

Abstract A 10-year-old spayed female Maltese with a history of vomiting and lethargy was referred to the hospital. Physical examination revealed dehydration and severe pain following abdominal palpation. A large mass was observed in the cranial abdomen through radiography and ultrasonography. Laparotomy was performed to find the origin of the mass. The mass was about $8 \mathrm{~cm}$ originating from the cecum and subsequently removed. Histopathologic evaluation revealed that the cecal mass was suspected to be a mesenchymal-derived tumor. Through immunohistochemistry, the mass was diagnosed as a gastrointestinal stromal tumor (GIST) based on the c-kit expression. Given its recurrence, postoperative preventive therapy was initiated with masitinib mesylate, which is a tyrosine kinase inhibitor. The animal did not show any side effects during the medication period. After 6 months of therapy, it was well controlled without any recurrence. In this case, we introduced a novel postoperative management of GIST using masitinib mesylate.

Key words gastrointestinal stromal tumor, c-kit gene, postoperative management, masitinib mesylate. 


\section{Introduction}

Gastrointestinal tumor is an uncommon case of canine tumor with epithelial, neuroendocrine, hematopoietic or mesenchymal origin $(6,15)$. Gastrointestinal stromal tumors (GISTs) are a specific group of mesenchymal tumors, which occur mainly in the interstitial of Cajal cell (ICC) (19). GISTs have been reported in human, dogs, and many other species $(8,19,21)$. GISTs usually have nonspecific symptoms that are indistinguishable from other diseases like anorexia, lethargy, vomiting, abdominal pain, weight loss etc. (19). Likewise, it is hard to find a specific feature of GIST during blood test (19). Using advanced diagnostic imaging evaluation, GIST can be detected easily $(10,11,19)$. Immunohistochemistry (IHC) targeting c-kit, smooth muscle actin (SMA) is essential to diagnose the GIST.

The best treatment option for GIST is complete resection keeping sufficient free margins and avoiding tumor rupture (19). Recent reports have suggested that if tumor is unresectable or there is a possibility of metastasis, tyrosine kinase inhibitors (TKIs) can be considered as treatment options $(2,5,12)$. The types of TKI used in veterinary medicine include toceranib, imatinib, and masitinib. Masitinib is the first line of treatment for canine mast cell tumor (CMCT) and is effective for human GISTs (17).

In this case, there was a mass originating from the cecum which was diagnosed as GIST using IHC following biopsy. After surgical resection, masitinib was prescribed for postoperative management. We introduced masitinib for postoperative management in a case of GIST which is an indistinguishable tumor in the gastrointestinal tract.

\section{Case Report}

A 10-year-old spayed female Maltese, weighing $6.2 \mathrm{~kg}$, was presented with a history of vomiting and lethargy to a local animal hospital. The owner reported that the dog underwent severe vomiting and had dark red feces. Physical examination revealed a deep pink-colored mucous membrane, elongated capillary refill time, and severe pain with abdominal palpation. Urinalysis did not show any remarkable findings. A complete blood count (CBC) test revealed high levels of red blood cells (RBC) $\left(11.61 \times 10^{6} / \mu \mathrm{L}\right.$; normal range, 5.65-8.87 million cells/ $\mu \mathrm{L})$, and hematocrit (HCT) $(70.7 \%$; normal range, 37.3-61.7\%), and a low level of mean corpuscular volume (MCV) (60.9 fL; normal range, 61.6-73.5 fL). A serum biochemical profile revealed high levels of alkaline phosphatase (ALP) (497 IU/L; normal range, 47-254 IU/L), aspartate transaminase (AST) (53 U/L; normal range, 17-44 $\mathrm{U} / \mathrm{L})$, ammonia $(110 \mu \mathrm{g} / \mathrm{dL}$; normal range, $16-75 \mu \mathrm{g} / \mathrm{dL})$, total bilirubin (1.2 mg/dL; normal range, 0.1-0.5 mg/dL), blood urea nitrogen $(42.6 \mathrm{mg} / \mathrm{dL}$; normal range, $9.2-29.2 \mathrm{mg} / \mathrm{dL}$ ), total protein $(8.3 \mathrm{~g} / \mathrm{dL}$; normal range, 5.0-7.2 g/dL), albumin (4.4 g/dL; normal range, 2.6-4.0 g/dL). An electrolyte test displayed the hyponatremia $(136 \mathrm{mEq} / \mathrm{L}$; normal range, 145 $151 \mathrm{mEq} / \mathrm{L}$ ) and hypokalemia (2.8 mEq/L; normal range, 3.9$5.1 \mathrm{mEq} / \mathrm{L})$ levels.

Radiological examination revealed the presence of an abnormal mass with soft tissue opacity in the cranial abdomen and ultrasonography revealed a hypoechoic mass near the duodenum (Fig. 1). Further, hyperechogenicity was detected near the pancreas suspected due to occurrence of ascite. Laparotomy was performed to find the origin of the abdominal mass. Acepromazine $(0.02 \mathrm{ml} / \mathrm{kg}$ ) (Sedaject Injection, SAMU MEDIAN) and butorphanol $(0.1 \mathrm{ml} / \mathrm{kg})$ (BUTOPHAN Injection, Myungmoon Pharm) were used as premedication for anesthesia followed by propofol (5 mg/kg) (Provive Inj. $1 \%$, Myungmoon Pharm) used for induction. Anesthesia was maintained with isoflurane (Ifran, Hana Pharm). During median incision, a rupture in the cecum was observed with
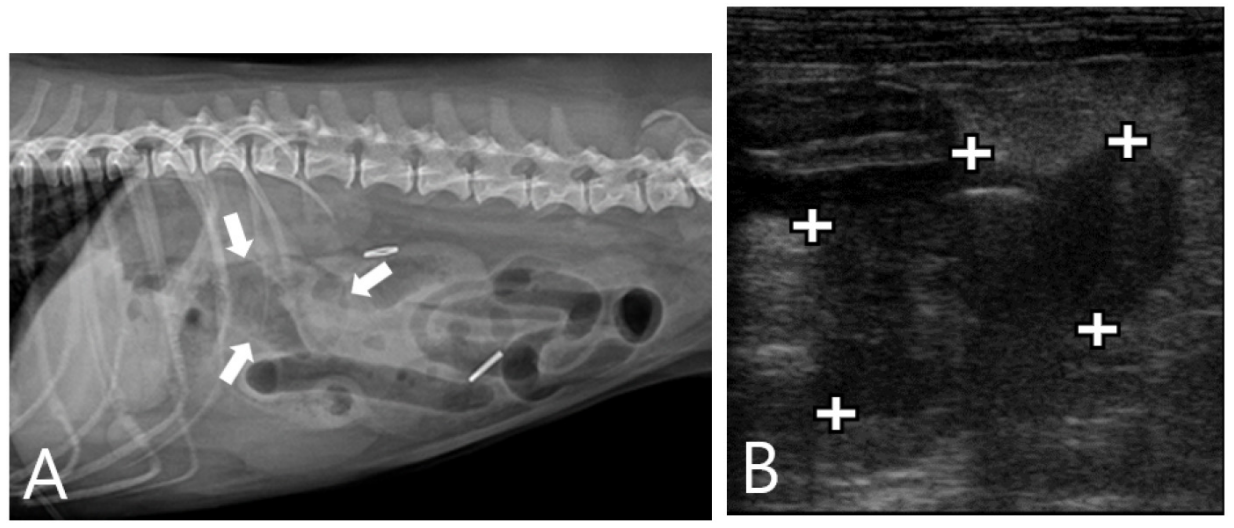

Fig. 1. (A) Abdominal radiographs of the dog (lateral view). A mass is identified in the cranial abdomen (arrow sign). The structure has soft tissue opacity causing mild cranial deviation of the liver. (B) Abdominal ultrasonography of the dog. A mass with irregular margin is detected (+ sign) near the duodenum. Large hypoechoic structure distinct to hyperechogenicity of mesenteric fat is detected. 

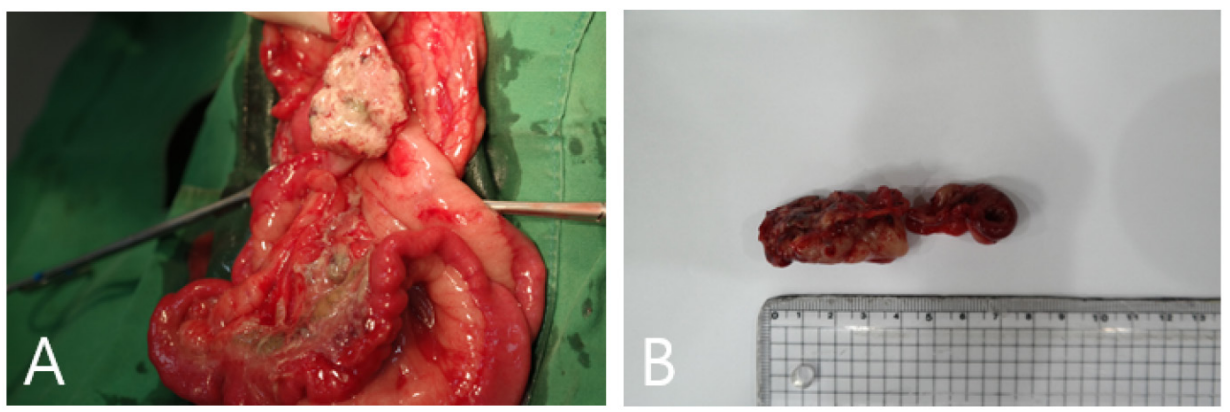

Fig. 2. Cecumectomy. (A) The mass shows severe purulent inflammations and necrosis of intestinal tissue. Suppurative exudates are observed prominently. (B) The surface of the mass excised from cecum is irregular and its size is approximately $8 \mathrm{~cm}$.
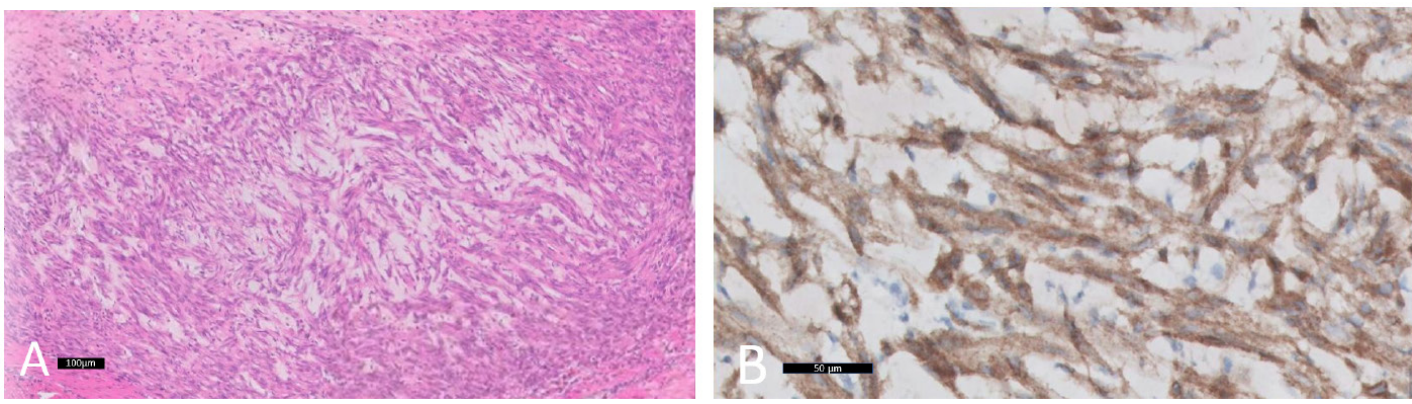

Fig. 3. Gastrointestinal stromal tumor in a dog's cecum. (A) An unencapsulated, loosely cellular, infiltrative mesenchymal neoplasm markedly expands to the intestinal muscular layer. The tumor is composed of interlacing bundle of spindloid cells disorderedly arranged. Spindloid cells are often separated by small amount of collagenous matrix and basophilic material with marked anisocytosis and anisokaryosis. (H\&E stain, $\times 100$, and Bar $=$ $100 \mu \mathrm{m}$ ). (B) Immunohistochemistry (IHC) revealed positive reaction (brown color) in mesenchymal-derived cells of the tumor (IHC for c-kit protein, $\times 200$, and $\mathrm{Bar}=50 \mu \mathrm{m})$.

suppurative ascite (Fig. 2A). Peritoneal lavage was performed simultaneously during surgery. The neoplasm accompanied by severe purulent inflammation and necrosis of the intestinal tissue, was removed by cecumectomy (Fig. 2B). The removed tissue from the tumor was subjected to histopathological analysis.

Histopathologic examination revealed an unencapsulated, infiltrative, expansile, loosely cellular neoplasm presented in the intestinal muscular wall (Fig. 3A). Further, spindloid cells were found arranged in short interlacing bundles and streams at random. Marked anisokaryosis was observed as prominent and multiple nucleoli and coarse aggregation of chromatin as well as a high mitotic index (6 per 10 high power fields) were detected. In central cavitation, multiple areas of necrosis and hemorrhage were noted. Overall, the cecal mass was diagnosed as either leiomyosarcoma or GIST through histopathology. Thus, immunohistochemical evaluation was necessary to confirm the diagnosis.

Approximately $60-70 \%$ neoplastic cells were found to be cytoplasmic positive for c-kit at moderate to strong degree through IHC (Fig. 3B). Approximately 30-40\% cells were moderately cytoplasmic positive for SMA. Consequently, immunohistochemical evaluation confirmed the definitive diag- nosis of tumor as GIST.

Considering the tumor-biological characteristics of GIST, preventive therapy using masitinib mesylate (Masivet, $A B$ science) was commenced postoperatively. Two weeks following surgical resection, administration of masitinib $(10 \mathrm{mg} / \mathrm{kg} /$ day) was initiated with famotidine $(0.5 \mathrm{mg} / \mathrm{kg} /$ day) (Famotidine tablet, Nelson) and liver protectants. Regular checkup was continued every 2 weeks for the first 2 months, and subsequently once every month. During the period of monitoring, no side effects were reported and the administration of the drugs was stopped after 24 weeks of treatment. Since then, we followed up the patient for 12 months and there has been no recurrence of GIST during that period.

\section{Discussion}

GISTs arise from the ICC which have characteristics of both smooth muscle and neural differentiation (13). GISTs have atypical histological features and diverse differentiations (19). In the past, GIST was misinterpreted as leiomyosarcoma or leiomyoma because of similar histological features (16). However, recent studies have reclassified these tumors as GIST by using $\mathrm{IHC}$ (16). 
Like other tumors, there is no specific clinical sign in GIST (19). In CBC, high levels of RBC and HCT seemed to be the result of dehydration. Results of biochemical tests suggested an injury of the liver with abnormality in its function. However, there was no evidence of a suspected metastasis in image examination. Other reports have indicated a possibility of anemia and leukocytosis accompanying GISTs (4). Anemia occurs due to intestinal injury caused by the increasing size of GIST. In this case, dehydration associated with vomiting appeared to have masked the symptoms of anemia.

Ultrasonography is the best way to identify the tumor origin and to predict metastasis $(4,11)$. Although it cannot distinguish GIST from non-GIST, ultrasonographic evaluation can be helpful to determine the extent of involvement for gastrointestinal tumors (11). Furthermore, abdominal effusion is a prominent sign of GIST which can be found by ultrasound (11). In the present case, intestinal neoplasm was suspected and ascite was detected using ultasonography. These findings could make veterinarians perform laparotomy for resection of the tumor more effectively. For more advanced surgical approach, computed tomography could be recommended $(4,19)$. Unfortunately, in this case, it was not performed as the client had a burden of cost.

The definitive diagnosis of GIST was based on IHC (19). In IHC, the antibodies for vimentin, S100, desmin, SMA and c-kit are usually used $(3,4,18,20)$. SMA correlates with the activation of myofibroblasts. The expression of CD117, a c-kit protein, is a specific feature of the GIST. It distinguishes GIST from other mesenchymal tumors $(7,14,19)$. C-kit protein mediates stem cell factor signaling; mutations in the c-kit gene deform the c-kit protein and affect cell self-destruction or cell division, resulting in tumors (1). GISTs usually have a positive result for vimentin, c-kit and SMA but it does not react positively for desmin and S100 proteins (20). In contrast, leiomyomas are positive for SMA but negative for c-kit (20). In this case, as IHC results showed positive for both c-kit and SMA, the tumor could be differentiated from leiomyoma and finally diagnosed as a GIST.

Complete surgical resection is the most effective treatment for GIST $(4,19)$. If GISTs are unrecoverable or metastasized, administration of TKI should be one of the treatment options $(2,5,12,19)$. A recent study reported that a dog administered with imatinib mesylate achieved complete remission for unrecovered GIST and survived for long (12). Another report proved that a dog with metastatic GIST was treated successfully with toceranib (5).

Masitinib is a novel potent and selective TKI, targeting primarily mutated c-Kit receptor (17). It is the first anticancer therapy approved in veterinary medicine for the canine mast cell tumor (CMCT) treatment, harboring activating c-kit mutations (9). Considering its anti-proliferative action, masitinib is undergoing several clinical investigations for several human malignancies, especially in GISTs. Human GISTs showed high overall response-rate (complete and partial remission) and have the prolonged progression-free survival with masitinib compared to other TKIs (17).

Most canine GISTs are known to be associated with mutation in genes corresponding to tyrosine kinase receptor (19). Considering these pathophysiological features, there are several previous reports using TKI for the purpose of postoperative chemotherapy for canine GIST (2). A study reported no recurrence of GIST in four out of seven cases after postoperative administration of toceranib (2). Since masitinib is known to work with high potency and specificity compared with toceranib for malignant tumors, in the present case, we planned for therapeutic procedures with masitinib considering the recurrence characteristics of GIST (9). During the 24 weeks of masitinib administration in this animal, there was no evidence of recurrence or metastasis with any adverse effects. Regarding the overall results and prognosis, we speculated that masitinib is a type of TKI that could secure the quality of life with positive effects on recurrence compared with other TKIs. However, this study has a limitation. This being a single case, it is difficult to say definitively that masitinib allowed better prognosis than other kinds of TKI. Further clinical studies are needed in future to prove the efficacy of masitinib.

\section{Conclusions}

GISTs are mesenchymal tumors that occur in the gastrointestinal tract of dogs. Given its recurrence and metastasis rates, postoperative management is a possible option. Masitinib has not been used for canine GIST till now, but is commonly used for human GISTs and shows high safety and efficacy in other c-kit mutated tumor treatment. In this case, postoperative management using masitinib was successfully performed without any severe adverse effects or recurrence. Our study shows that administration of masitinib could be one of the therapeutic options for postoperative chemotherapy in canine GIST.

\section{Acknowledgements}

This work was supported by the National Research Foundation of Korea (NRF) grant funded by the Korea government (MSIT) (No. 2019R1F1A1060984). 


\section{Conflicts of Interest}

The authors have no conflicting interests.

\section{References}

1. Abbaspour Babaei M, Kamalidehghan B, Saleem M, Huri HZ, Ahmadipour F. Receptor tyrosine kinase (c-Kit) inhibitors: a potential therapeutic target in cancer cells. Drug Des Devel Ther 2016; 10 : 2443-2459.

2. Berger $E P$, Johannes $C M$, Jergens $A E$, Allenspach $K$, Powers BE, Du $Y$, et al. Retrospective evaluation of toceranib phosphate (Palla$\operatorname{dia}(\mathbb{R})$ use in the treatment of gastrointestinal stromal tumors of dogs. J Vet Intern Med 2018; 32: 2045-2053.

3. Choi J, Kim H, Lee H, Kim J, Yoon J. Medical imaging and immunohistochemical diagnosis of gastrointestinal stromal tumor originated from colon in a dog. Korean J Vet Res 2008; 48: 111-117.

4. de Veiga CSC, Baetas BPS, Nassif FBV, Pereira EAS, Wasques DG, Guedes PEB, et al. Gastrointestinal stromal tumor associated with hypertrophic osteopathy in a dog. Acta Scientiae Vet 2019; 47(Suppl 1): 464.

5. Elliott JW, Swinbourne F, Parry A, Baines L. Successful treatment of a metastatic, gastrointestinal stromal tumour in a dog with toceranib phosphate (Palladia). J Small Anim Pract 2017; 58: 416418.

6. Frgelecová L, Škorič M, Fictum P, Husník R. Canine gastrointestinal tract tumours: a restrospective study of 74 cases. Acta Vet Brno 2013; 82: 387-392.

7. Frost D, Lasota J, Miettinen M. Gastrointestinal stromal tumors and leiomyomas in the dog: a histopathologic, immunohistochemical, and molecular genetic study of 50 cases. Vet Pathol 2003; 40: 42-54

8. Gregory-Bryson E, Bartlett E, Kiupel M, Hayes S, Yuzbasiyan-Gurkan V. Canine and human gastrointestinal stromal tumors display similar mutations in c-KIT exon 11. BMC Cancer 2010; 10: 559.

9. Hahn KA, Ogilvie G, Rusk T, Devauchelle P, Leblanc A, Legendre $A$, et al. Masitinib is safe and effective for the treatment of canine mast cell tumors. J Vet Intern Med 2008; 22: 1301-1309.

10. Hanazono K, Fukumoto S, Hirayama K, Takashima K, Yamane Y, Natsuhori $\mathrm{M}$, et al. Predicting metastatic potential of gastrointestinal stromal tumors in dog by ultrasonography. J Vet Med Sci.
2012; 74: 1477-1482.

11. Hobbs J, Sutherland-Smith J, Penninck D, Jennings S, Barber L, Barton B. Ultrasonographic features of canine gastrointestinal stromal tumors compared to other gastrointestinal spindle cell tumors. Vet Radiol Ultrasound 2015; 56: 432-438.

12. Irie M, Takeuchi Y, Ohtake Y, Suzuki H, Nagata N, Miyoshi T, et al. Imatinib mesylate treatment in a dog with gastrointestinal stromal tumors with a c-kit mutation. J Vet Med Sci 2015; 77: 15351539.

13. Kumagai K, Uchida K, Miyamoto T, Ushigusa T, Shinohara S, Yamaguchi $R$, et al. Three cases of canine gastrointestinal stromal tumors with multiple differentiations and c-kit-expression. J Vet Med Sci 2003; 65: 1119-1122.

14. LaRock RG, Ginn PE. Immunohistochemical staining characteristics of canine gastrointestinal stromal tumors. Vet Pathol 1997; 34: 303-311.

15. Liptak JM, Withrow SJ. Tumors of the gastrointestinal tract. In: Withrow SJ Vail D, editors. Withrow and MacEwen's small animal clinical oncology. Missouri: Saunders Elsevier. 2007: 455-510.

16. Maas CP, ter Haar G, van der Gaag I, Kirpensteijn J. Reclassification of small intestinal and cecal smooth muscle tumors in 72 dogs: clinical, histologic, and immunohistochemical evaluation. Vet Surg 2007; 36: 302-313.

17. Marech I, Patruno R, Zizzo N, Gadaleta C, Introna M, Zito AF, et al. Masitinib (AB1010), from canine tumor model to human clinical development: where we are? Crit Rev Oncol Hematol 2014; 91 : 98-111.

18. Miettinen M, Lasota J. Gastrointestinal stromal tumors--definition, clinical, histological, immunohistochemical, and molecular genetic features and differential diagnosis. Virchows Arch 2001; 438: 1-12.

19. Montañés I, Vila A, Roura X, Santos L, Canturri A, Verdés J, et al. Gastrointestinal stromal tumors (GIST): retrospective study of 6 dogs. Clin Vet Peq Anim 2019; 39: 155-161.

20. Russell KN, Mehler SJ, Skorupski KA, Baez JL, Shofer FS, Goldschmidt $\mathrm{MH}$. Clinical and immunohistochemical differentiation of gastrointestinal stromal tumors from leiomyosarcomas in dogs: 42 cases (1990-2003). J Am Vet Med Assoc 2007; 230: 13291333.

21. Suwa A, Shimoda T. Intestinal gastrointestinal stromal tumor in a cat. J Vet Med Sci 2017; 79: 562-566 